\title{
COMPARAÇÃO DA PERCEPÇÃO TEMPORAL DE DOÇURA, AMARGOR E SABOR DE FRUTA EM SUCO DE MANGA RECONSTITUÍDO E ADOÇADO COM SACAROSE, MISTURA CICLAMATO/SACARINA 2:1, ASPARTAME, SUCRALOSE E ESTÉVIA
}

\author{
DANIELA CARDOSO UMBELINO CAVALLINI* \\ HELENA MARIA ANDRÉ BOLINI**
}

\begin{abstract}
Foi realizada análise tempo-intensidade dos estímulos doce, amargo e sabor de fruta em suco de manga, adoçado com quatro edulcorantes (ciclamato/sacarina 2:1, aspartame, sucralose e estévia) e com sacarose. A amostra adoçada com estévia apresentou a maior intensidade máxima para os estímulos doce e amargo e essa característica persistiu por longo período, indicando a presença de doçura e de amargor residual intenso. A amostra com sacarose caracterizou-se pelo gosto doce limpo, sem residual amargo. Em relação ao estímulo sabor de fruta, a sacarose exibiu a maior intensidade máxima. Já o aspartame apresentou o maior tempo total de duração do estímulo, indicando potencialização do sabor de fruta por esse edulcorante em suco de manga. O aspartame foi o edulcorante, cujo comportamento sensorial mais se aproximou ao da sacarose na análise tempo-intensidade.
\end{abstract}

PALAVRAS-CHAVES: MANGA - SUCO; ANÁLISE SENSORIAL; EDULCORANTE; ANÁLISE TEMPO-INTENSIDADE.

\section{INTRODUÇÃO}

A análise tempo-intensidade é definida como a medida da duração, velocidade e intensidade produzida por um único estímulo (AMERINE, PANGBORN e ROESSLER, 1965).

O primeiro estudo realizado para avaliar as características temporais de produtos alimentícios foi conduzido por NEILSON (1957), o qual

* Doutora em Alimentos e Nutrição, Departamento de Alimentos e Nutrição, Faculdade de Engenharia de Alimentos (FEA), Universidade Estadual de Campinas (UNICAMP), Campinas, SP, Brasil (e-mail:daniducavallini@ig.com.br). Professor Associado, Departamento de Alimentos e Nutrição, FEA, UNICAMP, Campinas, SP, Brasil (e-mail: hellini@fea.unicamp.br). 
demonstrou que o mesmo gosto ou aroma é percebido em diferentes intensidades ao longo de determinado período.

Existem poucos relatos sobre a utilização da técnica tempo-intensidade até 1970 (LEE e PANGBORN, 1986). Porém, com o avanço da informática e a possibilidade de desenvolvimento de programas específicos, a análise tempo-intensidade foi incorporada à avaliação sensorial de rotina para alimentos e bebidas.

Não existe consenso entre os pesquisadores sobre quais os parâmetros das curvas obtidas devem ser empregados na avaliação tempo-intensidade, mas muitos utilizam a área total sob a curva (Área), o tempo total de duração do estímulo em segundos (Ttot) e a intensidade máxima do estímulo (Imax) (DUBOIS e LEE, 1983; HARRISON e BERNHARDT, 1984; KEMP e BIRCH, 1992; KETELSEN, KEAY e WIET, 1993; MATUSZEWSKA e BARYLKOPIKIELAN, 1995; OTT, EDWARDS e PALMER, 1991; SWARTZ, 1980).

A manga figura entre as frutas tropicais com maior expressão econômica nos mercados nacional e internacional. Destaca-se pela aparência exótica, sabor e aroma agradáveis, e por tratar-se de boa fonte de carotenóides e de carboidratos (SEATON, 1996).

O Brasil ocupa o nono lugar entre os países produtores de manga, com área cultivada de 68 mil hectares e produção anual de 925.018 toneladas (IBGE, 2003). A fruta destina-se ao consumo direto e para industrialização na forma de compotas, geléias, sorvetes, néctares, polpas congeladas e sucos concentrados, que devem ser reconstituídos e adoçados antes do consumo.

A sacarose constitui a substância mais utilizada como adoçante de bebidas devido às suas características únicas de sabor. Porém, a preocupação com a saúde aumentou a procura por adoçantes não-calóricos, utilizados em substituição à sacarose, que auxiliam na redução e manutenção do peso corporal, no controle do diabetes e na prevenção da cárie dental (VERMUNT et al., 2003).

Segundo a Associação Brasileira da Indústria de Alimentos Dietéticos e para Fins Especiais (ABIAD), o mercado brasileiro de alimentos "diet" e "light" cresceu cerca de $870 \%$ nos últimos dez anos. Os produtos que mais contribuíram para esse crescimento foram os refrigerantes, os sucos de frutas e os adoçantes de mesa (ABIAD, 2004).

Os edulcorantes compreendem o grupo de substâncias, utilizadas em 
substituição à sacarose, que compartilham a propriedade de interagir com receptores gustativos e produzir a sensação percebida e denominada de doce (MONTIJANO, TOMÁS-BARBERÁN e BORREGO, 1998). Idealmente, o edulcorante deve apresentar perfil de sabor e propriedades funcionais semelhantes às da sacarose.

A caracterização temporal de dulçor de substâncias edulcorantes é importante para prever sua aceitação, pois cada uma apresenta características específicas de intensidade, persistência do gosto doce, presença de gosto residual e intensificação do sabor de fruta, que variam em função do alimento ao qual são adicionadas (CARDELLO, SILVA e DAMÁSIO, 1999; KETELSEN, KEAY, e WIET, 1993; MATYSIAK e NOBLE, 1991; WISEMAN e McDANIEL, 1991).

Considerando a crescente procura por bebidas de sabor diferenciado e a expansão do segmento de produtos com valores calóricos reduzidos, o estudo do comportamento sensorial do suco de manga adoçado com diferentes edulcorantes mostrou-se oportuno. Este trabalho teve por objetivo comparar a percepção temporal de doçura, amargor e sabor de fruta em suco de manga adoçado com sacarose, ciclamato/sacarina 2:1, aspartame, sucralose e estévia.

\section{MATERIAL E MÉTODOS}

\subsection{MATERIAL}

Foram utilizados suco de manga concentrado na forma líquida, marca comercial Maguary (Fleischmann Royal/ Nabisco), sacarose p.a. (Sigma Chemical Co.), mistura ciclamato/ sacarina na proporção de 2:1 da marca comercial Assugrin (Vepê Indústria Alimentícia Ltda), aspartame 100\% (Ajinomoto Co., Inc.), sucralose (Johnson \& Johnson) e extrato de folhas de estévia, com a seguinte composição: esteviosídeo - 81,0\%, rebaudiosídeo C - $0,6 \%$ e rebaudiosídeo $A-17,7 \%$ (Stéviafarma do Brasil).

\subsection{MÉTODOS}

\subsubsection{Preparação das Amostras}

O suco de manga concentrado foi preparado diluindo-se uma parte de suco para duas partes de água mineral, segundo as instruções do fabricante. 
Cada amostra foi adoçada, no momento das análises, com 8,0\% de sacarose, concentração pré-determinada em teste de aceitação utilizando escala de ideal (CAVALLINI, 2005). Os edulcorantes foram adicionados em concentrações equivalentes a $8,0 \%$ de sacarose, determinadas pelo teste de estimação de magnitude. As concentrações de cada edulcorante foram as seguintes: $0,0358 \%$ da mistura ciclamato/sacarina $2: 1,0,0555 \%$ de aspartame, $0,0159 \%$ de sucralose e $0,0850 \%$ de estévia.

As amostras foram preparadas com uma hora de antecedência, mantidas a $6 \pm 2^{\circ} \mathrm{C}$ e servidas (para avaliação sensorial) em temperatura de aproximadamente $10 \pm 2^{\circ} \mathrm{C}$. As avaliações sensoriais foram realizadas em cabines individuais e as amostras apresentadas em copos plásticos codificados com algarismos de três dígitos.

\subsubsection{Análise Sensorial}

Realizou-se a coleta de dados da análise tempo-intensidade pelo programa Sistema de Coleta de Dados Tempo-Intensidade (SCDTI), desenvolvido no Laboratório de Análise Sensorial da Faculdade de Engenharia de Alimentos da Universidade Estadual de Campinas (UNICAMP) (CARDELLO et al., 2003). Os estímulos doce, amargo e sabor de fruta foram avaliados separadamente.

\subsubsection{Seleção da Equipe de julgadores}

Efetuou-se a pré-seleção dos julgadores para a análise tempo-intensidade mediante testes triangulares de diferença, aplicados à análise seqüencial, e pela habilidade de trabalhar com o computador (AMERINE, PANGBORN e ROESSLER, 1965; MEILGAARD, CIVILLE e CARR, 1999). Os julgadores pré-selecionados participaram de várias sessões de treinamento para entendimento e padronização da metodologia.

Na etapa de seleção, 10 julgadores pré-selecionados realizaram a análise tempo-intensidade com amostras de suco de manga, adoçadas com os edulcorantes estudados a 8,0\% de equivalência de doçura em relação à sacarose. A seleção dos julgadores para análise tempo-intensidade do estímulo doce foi efetuada com amostras de suco de manga adoçadas com sacarose e com a mistura ciclamato/sacarina 2:1. Para o estímulo amargo o suco foi adoçado com mistura ciclamato/sacarina 2:1 e estévia, e para o sabor de manga com sacarose e aspartame. A escolha dos edulcorantes utilizados na seleção dos julgadores baseou-se nas características principais de cada substância, verificadas em dados de literatura. As amostras foram apresentadas de forma monádica com três 
repetições e avaliadas separadamente.

A seleção dos julgadores foi realizada em função do poder de discriminação, repetibilidade e concordância com a equipe (DAMÁSIO e COSTELL, 1991). Os julgadores que obtiveram valores de $F_{\text {amostra }}$ significativo $(p<0,30), F_{\text {repeticăo }}$ não-significativo $(p>0,05)$ e $F$ interação amostra $x$ julgador não-significativo $(p>0,05)$ em relação a cada parâmetro da curva foram selecionados.

No total foram selecionados 8 julgadores que passaram por várias sessões de treinamento antes da realização dos testes finais.

\subsubsection{Avaliação das Amostras}

$\mathrm{Na}$ análise tempo-intensidade as amostras foram apresentadas de forma monádica e aleatória, com três repetições, em copos plásticos codificados com algarismos de três dígitos. Padronizou-se a quantidade de amostra em $5 \mathrm{~mL}$ por teste, sendo os julgadores instruídos a colocar o volume total da amostra na boca e a seguir as instruções dadas pelo programa.

Cada sessão foi iniciada pela escolha da opção mostrada na tela do computador, mediante pressão no "mouse". Ao primeiro aviso sonoro emitido, o julgador colocava toda a amostra na boca, ao segundo aviso o engolia e o terceiro aviso determinava o final do teste. A cada etapa o julgador indicava na escala, usando o "mouse", a intensidade do atributo sensorial avaliado.

Padronizou-se o tempo de espera em cinco segundos, tempo de retenção antes da ingestão em cinco segundos e tempo de análise após a ingestão em um minuto e trinta segundos para a doçura, dois minutos para o amargor e um minuto para o sabor de manga. Utilizou-se escala estruturada de nove pontos no teste, cujo 0 corresponde a nenhum, 4,5 a moderado e 9,0 a forte.

Após a coleta de dados em cada sessão de avaliação sensorial, o programa analisa e fornece os seguintes parâmetros: intensidade máxima registrada pelo julgador (Imax), tempo para atingir a intensidade máxima (Timax), tempo total de duração do estímulo (Ttot), área sob a curva tempo-intensidade (Área), tempo para início da percepção do estímulo (Ti) e tempo final da percepção do estímulo (Tf) .

\subsubsection{Análise dos Dados}

Os dados dos parâmetros mais importantes da curva foram submetidos à análise de variância (ANOVA) de dois fatores (amostra e julgadores) e 
interação para cada parâmetro. Aplicou-se o teste de Tukey para comparação das médias, sendo também efetuada a Análise de Componentes Principais. Todas as análises foram feitas utilizando-se o programa para análise estatística SAS (2001).

\section{RESULTADOS E DISCUSSÃO}

\subsection{ANÁLISE TEMPO-INTENSIDADE PARA O ESTÍMULO DOCE}

A partir dos parâmetros coletados para cada amostra e cada julgador foi realizada a análise de componentes principais $(\mathrm{ACP})$, cujo resultado está apresentado na Figura 1.

Verificou-se que $76,03 \%$ da variação ocorrida entre as amostras foi explicada pelo primeiro eixo (Componente Principal 1), sendo que todos os parâmetros Timax, Ttot, Área, Ti e Tf (positivamente) contribuíram de forma praticamente equivalente para a variabilidade associada a esse eixo. O atributo Imax (positivamente) foi o que mais contribuiu para a variabilidade associada ao segundo eixo (Componente Principal 2). As amostras apresentaram boa repetibilidade.

A sacarose evidenciou os menores valores em todos os parâmetros das curvas tempo-intensidade. A mistura ciclamato/sacarina 2:1, o aspartame e a sucralose caracterizaram-se, principalmente, pelo parâmetro Imax. A estévia apresentou comportamento distinto das demais amostras, sendo caracterizada pelos atributos Ti, Timax, Tf, Ttot e Área.

A proximidade dos vetores da ACP indicou a possibilidade de correlação positiva entre Timax, Tf, Ttot e área para o estímulo doce. Os valores dos coeficientes de correlação linear de Pearson (r), apresentados na Tabela 1 confirmaram essa observação.

\section{TABELA 1 - VALORES DOS COEFICIENTES DE CORRELAÇÃO LINEAR DE PEARSON (R) ENTRE OS VETORES DA ANÁLISE DE COMPONENTES PRINCIPAIS PARA O ESTÍMULO DOCE EM SUCO DE MANGA}

\begin{tabular}{lcccccc}
\hline Vetores & Imax & Timax & Ttot & Area & Ti & Tf $^{*}$ \\
\hline Imax & 1,00000 & & & & & \\
Timax & 0,25164 & 1,00000 & & & & \\
Ttot & 0,38889 & 0,96695 & 1,00000 & & & \\
Área & 0,56579 & 0,86264 & 0,95077 & 1,00000 & & \\
Ti & $-0,12959$ & 0,83583 & 0,72509 & 0,51668 & 1,00000 & \\
Tf & 0,35594 & 0,97379 & 0,99807 & 0,94444 & 0,75032 & 1,00000 \\
\hline
\end{tabular}

*Imax = intensidade máxima, Timax = tempo para atingir a intensidade máxima, Ttot = tempo total de duração do estímulo, Area = área sob a curva, Ti = tempo de início da percepção do estímulo, $\mathrm{Tf}=$ tempo final da percepção do estímulo. 
FIGURA 1 - GRÁFICO BIDIMENSIONAL DA ACP DOS PARÂMETROS DAS CURVAS TEMPO-INTENSIDADE PARA O ESTÍMULO DOCE DAS AMOSTRAS DE SUCO DE MANGA, ADOÇADAS COM SACAROSE (SAC), CICLAMATO/ SACARINA 2:1 (C/S 2:1), ASPARTAME (ASP), SUCRALOSE (SUC) E ESTÉVIA (EST) A 8,0\% DE EQUIVALÊNCIA DE DOÇURA

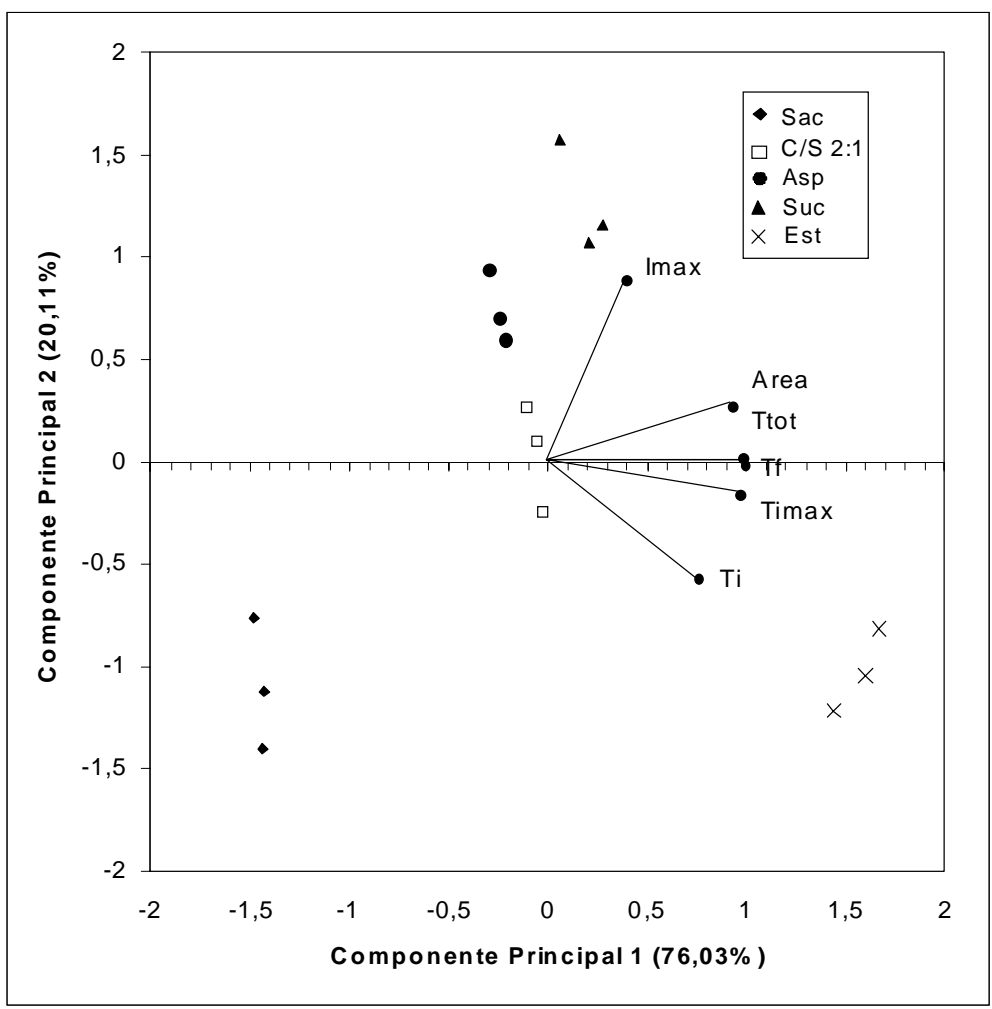

A análise de variância (ANOVA) evidenciou diferença significativa $(p<0,001)$ entre as amostras em relação a todos os parâmetros estudados.

A Tabela 2 mostra a comparação das médias das amostras para cada um dos parâmetros, obtidas pelo teste de médias de Tukey $(p \leq 0,05)$. As características das curvas tempo-intensidade registradas para o estímulo doce são apresentadas, graficamente, na Figura 2. Para a construção das curvas foram utilizadas as médias dos parâmetros de cada amostra. 


\section{TABELA 2 - MÉDIAS DOS PARÂMETROS DAS CURVAS TEMPO- INTENSIDADE PARA O ESTÍMULO DOCE DOS EDULCORANTES COM DOÇURA EQUIVALENTE A 8,0\% DE SACAROSE ADICIONADOS EM SUCO DE MANGA}

\begin{tabular}{ccccccc}
\hline Amostras & Imax & Timax & Ttot & Área & Ti & Tf \\
\hline Sac & $5,5003^{\mathrm{c}}$ & $8,4713^{\mathrm{c}}$ & $18,0163^{\mathrm{d}}$ & $54,6090^{\mathrm{d}}$ & $1,8625^{\mathrm{b}}$ & $19,8788^{\mathrm{a}}$ \\
C/S $2: 1$ & $5,8411^{\mathrm{b}}$ & $9,8238^{\mathrm{b}}$ & $25,8821^{\mathrm{b}}$ & $84,8330^{\mathrm{b}}$ & $1,8958^{\mathrm{b}}$ & $27,7793^{\mathrm{b}}$ \\
Asp & $6,1268^{\mathrm{a}}$ & $9,6454^{\mathrm{b}}$ & $23,9100^{\mathrm{c}}$ & $79,1710^{\mathrm{c}}$ & $1,9279^{\mathrm{b}}$ & $25,8379^{\mathrm{c}}$ \\
Suc & $6,1665^{\mathrm{a}}$ & $10,2179^{\mathrm{b}}$ & $27,2650^{\mathrm{b}}$ & $88,3990^{\mathrm{b}}$ & $1,7950^{\mathrm{b}}$ & $29,0600^{\mathrm{b}}$ \\
Est & $5,8377^{\mathrm{b}}$ & $12,6117^{\mathrm{a}}$ & $34,1800^{\mathrm{a}}$ & $96,9980^{\mathrm{a}}$ & $2,3633^{\mathrm{a}}$ & $36,5432^{\mathrm{a}}$ \\
\hline DMS $^{*}$ & 0,2283 & 1,0437 & 1,4142 & 4,3799 & 0,2183 & 1,3883 \\
\hline
\end{tabular}

${ }^{*}$ DMS = diferença mínima significativa obtida no teste de médias de Tukey. Médias iguais na mesma coluna não diferem significativamente entre si $(p>0,05)$. Imax = intensidade máxima, Timax = tempo para atingir a intensidade máxima, Ttot = tempo total de duração do estímulo, Área = área sob a curva, $\mathrm{Ti}=$ tempo de início da percepção do estímulo, $\mathrm{Tf}$ = tempo final da percepção do estímulo.

$\mathrm{SAC}=$ sacarose $, \mathrm{C} / \mathrm{S} 2: 1$ = ciclamato/sacarina, $\mathrm{Asp}=$ aspartame, $\mathrm{Suc}=$ sucralose Est $=$ estévia.

A sucralose e o aspartame apresentaram as maiores médias para o parâmetro Imax, sem revelar diferença significativa entre si $(p>0,05)$. A mistura ciclamato/sacarina 2:1 e a estévia (com médias intermediárias para Imax) não evidenciaram diferença significativa entre si, porém foram diferentes $(p<0,05)$ das demais amostras. A sacarose apresentou a menor média para o parâmetro intensidade máxima e diferiu significativamente das demais amostras.

Em relação ao Timax, a estévia apresentou a maior média e a sacarose a menor, diferindo significativamente das demais amostras. A sucralose, o aspartame e a mistura ciclamato/sacarina 2:1 apresentaram médias intermediárias, sem diferir significativamente entre si.

A curva do suco de manga adoçado com estévia evidenciou o maior tempo total de duração (Ttot), seguido pelas curvas da sucralose, da mistura ciclamato/ sacarina 2:1 e do aspartame. Já a curva da amostra adoçada com sacarose apresentou o menor tempo total de duração (Ttot), verificando-se diferença significativa $(p<0,05)$ entre todas as amostras 
para esse parâmetro.

As médias de Área obedeceram a seguinte ordem decrescente: estévia, sucralose, ciclamato/sacarina 2:1, aspartame e sacarose, sendo que somente a mistura ciclamato/sacarina 2:1 e a sucralose não diferiram significativamente entre si.

A estévia apresentou a maior média para Ti (tempo inicial de percepção do estímulo), diferindo significativamente das outras amostras que por sua vez não diferiram entre si.

FIGURA 2 - CURVAS TEMPO-INTENSIDADE DO ESTÍMULO DOCE PARA SACAROSE (SAC), CICLAMATO/SACARINA 2:1 (C/S 2:1), ASPARTAME (ASP), SUCRALOSE (SUC) E ESTÉVIA (EST) A 8,0\% DE EQUIVALÊNCIA DE DOÇURA EM SUCO DE MANGA

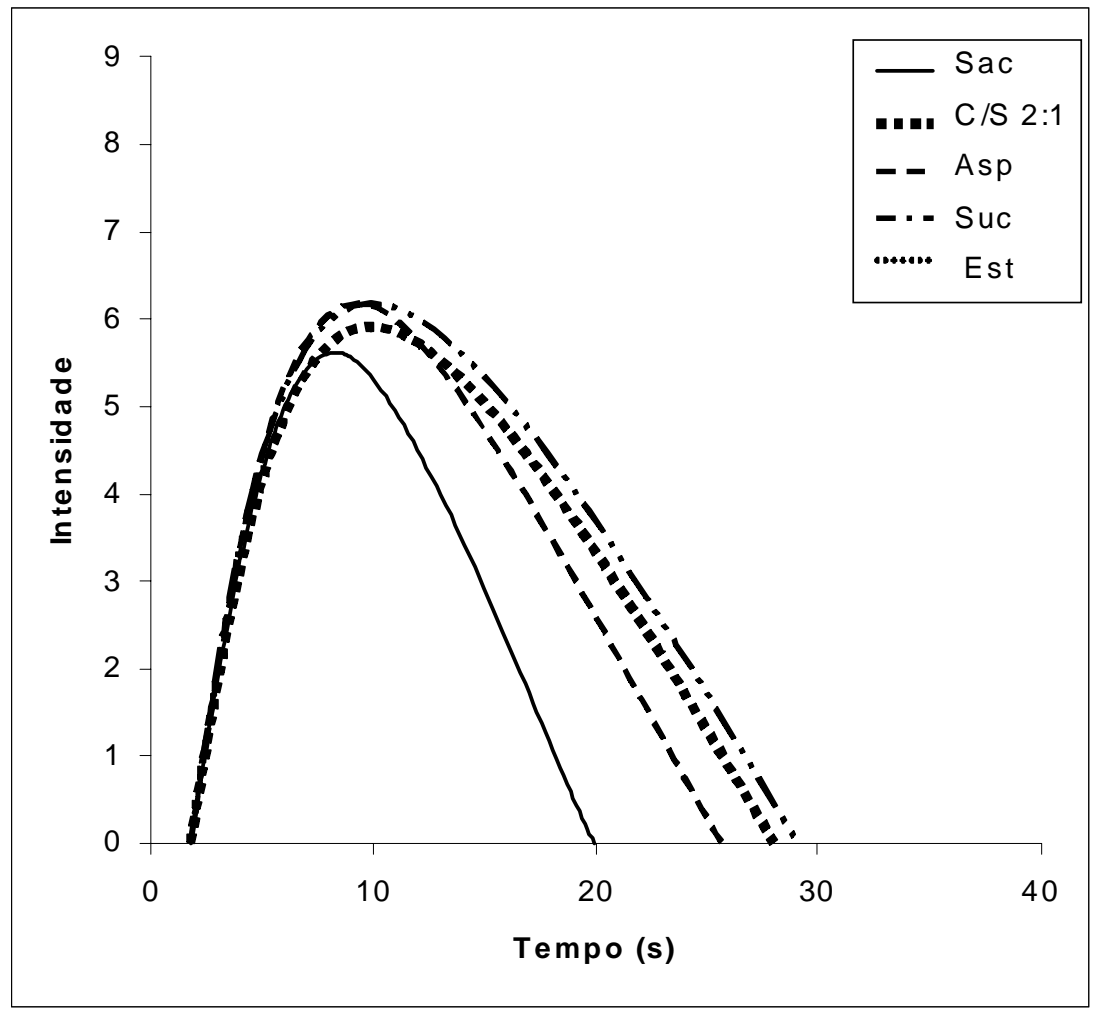


Para a maioria dos parâmetros estudados em relação ao estímulo doce, o aspartame foi o edulcorante cujo perfil temporal mais se aproximou ao da sacarose em suco de manga. A estévia apresentou o comportamento mais distinto em relação à sacarose, evidenciando tempo total de duração do estímulo (Ttot) e área sob a curva (Área) superiores.

LARSON-POWERS e PANGBORN (1978) realizaram análise tempointensidade de sacarose, aspartame, ciclamato e sacarina a $10 \%$ de equivalência de doçura em soluções aquosas e em bebidas sabor morango, laranja e limão. $O$ aspartame e a sacarose apresentaram curvas tempo-intensidade semelhantes.

CARDELLO (1996) avaliou o comportamento temporal para o estímulo doce de soluções aquosas de sacarose, aspartame, mistura ciclamato/ sacarina 2:1 e estévia a 10\% de equivalência de doçura. A amostra que mais se aproximou da sacarose foi o aspartame. A estévia apresentou maiores médias para Ttot e Área e diferiu em maior grau da sacarose.

\subsection{ANÁLISE TEMPO-INTENSIDADE PARA O ESTÍMULO AMARGO}

O resultado da análise de componentes principais para o estímulo amargo em suco de manga está representado na Figura 3.

Os componentes principais 1 e 2 explicaram juntos $99,56 \%$ da variação ocorrida entre as amostras. Verificou-se que $81,70 \%$ da variação foi explicada pelo primeiro eixo (CP1), sendo que os parâmetros Área, Ttot, Tf, Timax e Imax (positivamente) contribuíram de forma semelhante para a variabilidade associada a esse eixo. O parâmetro Ti (positivamente) foi o que mais contribuiu para a variabilidade associada ao segundo eixo (CP2).

O experimento apresentou ótima repetibilidade. A sacarose e o aspartame ficaram em regiões próximas no gráfico bidimensional, não sendo caracterizados por nenhum parâmetro específico. A sucralose e a mistura ciclamato/sacarina 2:1 também ficaram em regiões próximas e foram caracterizadas, principalmente, pelo Ti. A estévia apresentou comportamento distinto das demais amostras e caracterizou-se pelos parâmetros Área, Ttot, Imax, Tf e Timax.

Os valores dos coeficientes de correlação linear ( $r$ ) dos vetores da ACP (Tabela 3) confirmaram a elevada correlação linear positiva entre alguns dos parâmetros por eles representados. 
TABELA 3 - VALORES DOS COEFICIENTES DE CORRELAÇÃO LINEAR DE PEARSON (R) ENTRE OS VETORES DA ANÁLISE DE COMPONENTES PRINCIPAIS PARA $O$ ESTÍMULO AMARGO EM SUCO DE MANGA

\begin{tabular}{lcccccc}
\hline \multicolumn{1}{c}{ Vetores } & Imax & Timax & Ttot & Área & Ti & Tf $^{*}$ \\
\hline Imax & 1,00000 & & & & & \\
Timax & 0,84111 & 1,00000 & & & & \\
Ttot & 0,99947 & 0,82709 & 1,00000 & & & \\
Área & 0,99453 & 0,78699 & 0,99711 & 1,00000 & & \\
Ti & 0,30571 & 0,74771 & 0,28674 & 0,23376 & 1,00000 & \\
Tf & 0,99952 & 0,84649 & 0,99928 & 0,99440 & 0,32245 & 1,00000 \\
\hline
\end{tabular}

Imax = intensidade máxima, Timax = tempo para atingir a intensidade máxima, Ttot = tempo total de duração do estímulo, Área = área sob a curva, $\mathrm{Ti}=$ tempo de início da percepção do estímulo, Tf = tempo final da percepção do estímulo.

FIGURA 3-GRÁFICO BIDIMENSIONAL DA ACP DOS PARÂMETROS DAS CURVAS TEMPO-INTENSIDADE PARA O ESTÍMULO AMARGO DAS AMOSTRAS DE SUCO DE MANGA ADOÇADAS COM SACAROSE (SAC), CICLAMATO/ SACARINA 2:1 (C/S 2:1), ASPARTAME (ASP), SUCRALOSE (SUC) E ESTÉVIA (EST) A 8,0\% DE EQUIVALÊNCIA DE DOÇURA

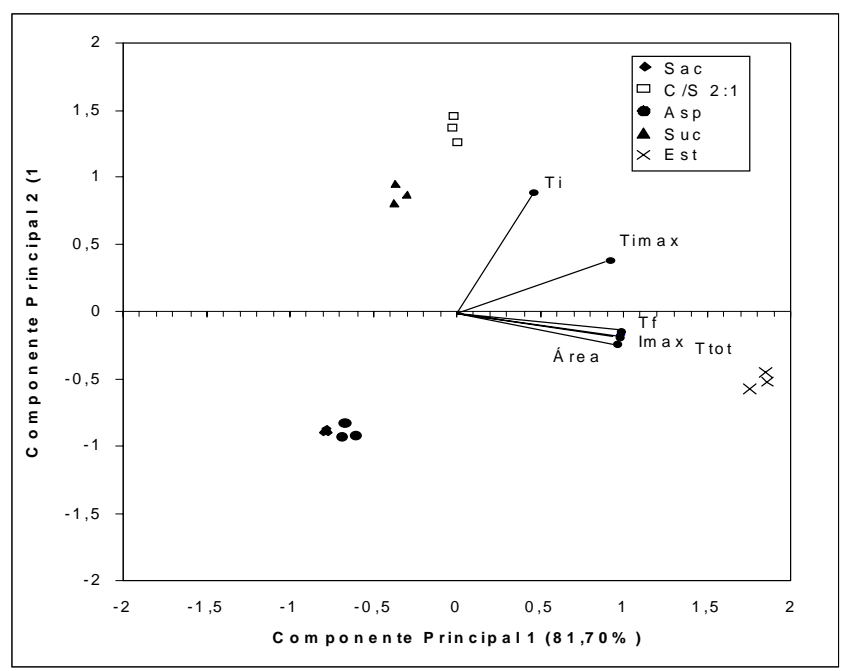

A análise de variância revelou diferença significativa $(p<0,001)$ entre as amostras em relação a todos os parâmetros estudados. A Tabela 4 expressa os resultados do teste de médias de Tukey para o estímulo amargo em suco de manga. 
A estévia apresentou a maior Imax, seguida pela mistura ciclamato/ sacarina 2:1, aspartame, sucralose e sacarose. Somente o aspartame e a sucralose não diferiram $(p>0,05)$ entre si em relação a esse parâmetro.

As amostras apresentaram diferença significativa entre si para os parâmetros Timax e Tf, sendo classificadas na seguinte ordem decrescente: estévia, mistura ciclamato/sacarina 2:1, sucralose, aspartame e sacarose.

O maior tempo total de duração do estímulo (Ttot) foi observado para a estévia, seguida pela mistura ciclamato/sacarina 2:1 e o menor para a sacarose (todos com diferença significativa entre si).

O parâmetro Ti obedeceu a seguinte ordem crescente: aspartame, sacarose, estévia, sucralose e mistura ciclamato/sacarina 2:1, sendo que somente os dois primeiros edulcorantes não diferiram significativamente entre si.

As amostras adoçadas com sacarose, sucralose e aspartame apresentaram as menores médias para o parâmetro Área e não diferiram entre si $(p>0,05)$. A estévia evidenciou a maior Área seguida pela mistura ciclamato/sacarina 2:1 e esses edulcorantes diferiram significativamente entre si e das demais amostras.

\section{TABELA 4 - MÉDIAS DOS PARÂMETROS DAS CURVAS TEMPO- INTENSIDADE PARA O ESTÍMULO AMARGO DOS EDULCORANTES COM DOÇURA EQUIVALENTE A 8,0\% DE SACAROSE, ADICIONADOS EM SUCO DE MANGA}

\begin{tabular}{ccccccc}
\hline Amostras & Imax & Timax & Ttot & Área & Ti & Tf \\
\hline Sac & $0,0830^{\mathrm{d}}$ & $2,5779^{\mathrm{e}}$ & $1,4554^{\mathrm{d}}$ & $0,2900^{\mathrm{c}}$ & $2,0721^{\mathrm{d}}$ & $3,5275^{\mathrm{e}}$ \\
C/S & $1,1106^{\mathrm{b}}$ & $7,5717^{\mathrm{b}}$ & $8,9754^{\mathrm{b}}$ & $6,2830^{\mathrm{b}}$ & $4,4433^{\mathrm{a}}$ & $13,3887^{\mathrm{b}}$ \\
Asp & $0,4271^{\mathrm{c}}$ & $3,5083^{\mathrm{d}}$ & $3,3896^{\mathrm{c}}$ & $1,8930^{\mathrm{c}}$ & $1,9771^{\mathrm{d}}$ & $5,3667^{\mathrm{d}}$ \\
Suc & $0,3652^{\mathrm{c}}$ & $5,3033^{\mathrm{c}}$ & $3,0850^{\mathrm{c}}$ & $1,6500^{\mathrm{c}}$ & $3,9417^{\mathrm{b}}$ & $7,0267^{\mathrm{c}}$ \\
Est & $7,0548^{\mathrm{a}}$ & $9,9408^{\mathrm{a}}$ & $67,3217^{\mathrm{a}}$ & $210,392^{\mathrm{a}}$ & $3,6538^{\mathrm{c}}$ & $70,9755^{\mathrm{a}}$ \\
\hline DMS & 0,0698 & 0,3012 & 1,1871 & 3,4359 & 0,2823 & 1,1450 \\
\hline
\end{tabular}

DMS = diferença mínima significativa obtida no teste de médias de Tukey.

Médias iguais na mesma coluna não diferem significativamente entre si $(p>0,05)$. Imax = intensidade máxima, Timax = tempo para atingir a intensidade máxima, Ttot $=$ tempo total de duração do estímulo, Área = área sob a curva, $\mathrm{Ti}=$ tempo de início da percepção do estímulo, Tf = tempo final da percepção do estímulo.

$\mathrm{SAC}=$ sacarose, C/S 2:1 = ciclamato/sacarina, Asp = aspartame, Suc = sucralose, Est $=$ estévia. 
A representação gráfica das médias dos parâmetros das curvas tempointensidade dos edulcorantes para o estímulo amargo em suco de manga está ilustrada na Figura 4.

FIGURA 4 - CURVAS TEMPO-INTENSIDADE DO ESTÍMULO AMARGO PARA CICLAMATO/SACARINA 2:1 (C/S 2:1), ASPARTAME (ASP), SUCRALOSE (SUC) E ESTÉVIA (EST) A 8\% DE EQUIVALÊNCIA DE DOÇURA EM SUCO DE MANGA

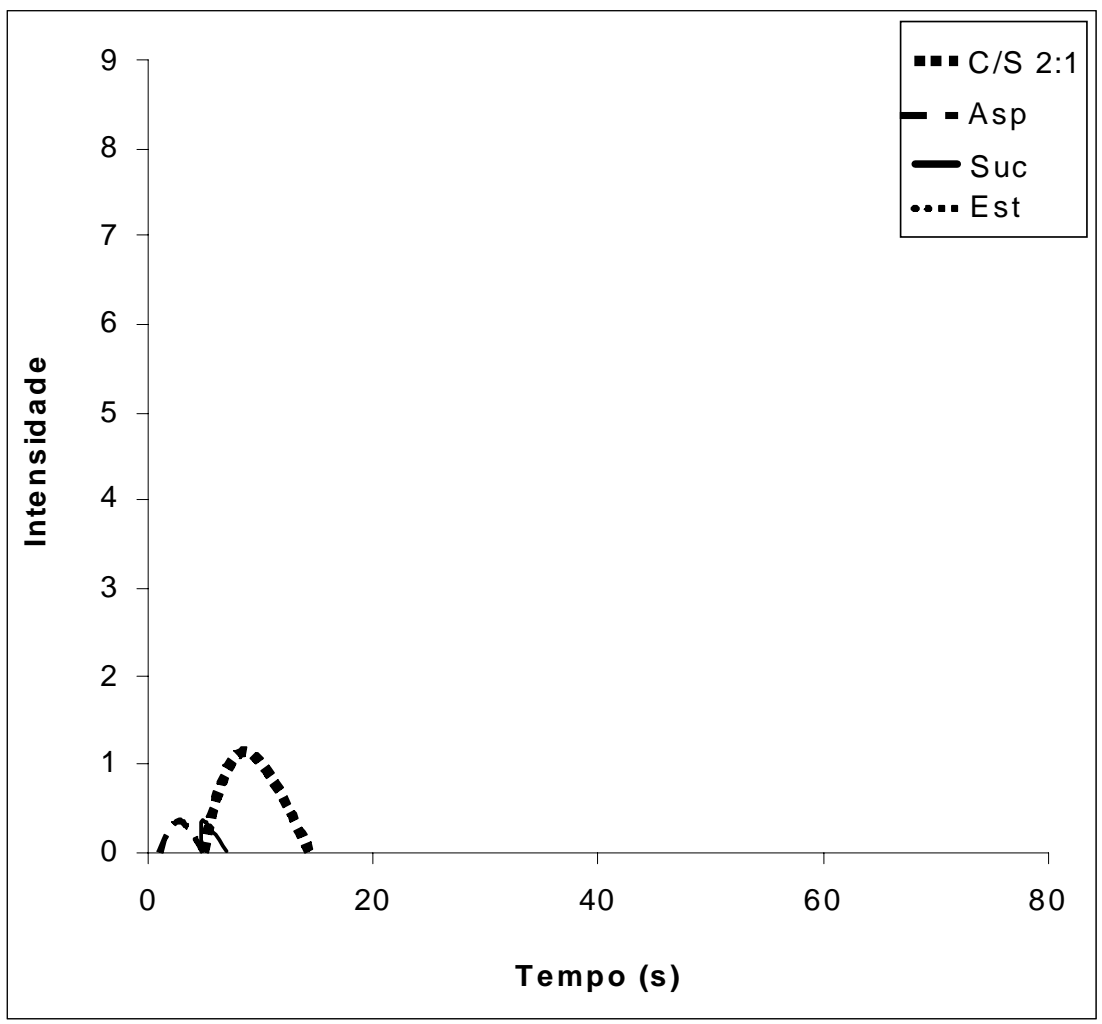

A sacarose exibiu médias baixas para todos os parâmetros, não sendo possível representá-la graficamente.

A estévia apresentou maior intensidade para o estímulo amargo em suco de manga, a qual persistiu por longo período. 
Em relação ao amargor, novamente, o aspartame foi o edulcorante que mais se aproximou da sacarose em suco de manga.

LARSON-POWERS e PANGBORN (1978) observaram que os perfis sensoriais da sacarose e do aspartame para o estímulo amargo (a 10\% de equivalência de doçura) eram indistinguíveis, enquanto o ciclamato e a sacarina apresentaram amargor superior. Outros pesquisadores verificaram que em solução aquosa a $10 \%$ de equivalência de doçura, 0 aspartame mostrou-se semelhante à sacarose para o estímulo amargo (OTT, EDWARDS e PALMER, 1991).

Segundo CARDELLO (1996) e CARDELLO, SILVA e DAMÁSIO (1999) a estévia a $10 \%$ de equivalência de doçura exibiu as maiores médias para os parâmetros Imax, Ttot, Área e Timax. O aspartame e a mistura ciclamato/sacarina 2:1 mostraram-se semelhantes entre si para os parâmetros Ttot, Imax e Área. Não foi registrada intensidade para o estímulo amargo para a sacarose. O aspartame foi o edulcorante que mais se aproximou da sacarose, apesar de não diferir significativamente $(p>0,05)$ da mistura ciclamato/sacarina 2:1 .

\subsection{ANÁLISE TEMPO-INTENSIDADE PARA O ESTÍMULO SABOR DE FRUTA}

A Figura 5 mostra os resultados da análise de componentes principais (ACP) para o estímulo sabor de fruta em suco de manga. Os componentes principais 1 e 2 explicaram juntos $91,85 \%$ da variação ocorrida entre as amostras. O primeiro eixo (CP1) explicou $65,03 \%$ da variação e os parâmetros Ttot, Tf, Área e Imax (positivamente) constituíram os principais responsáveis por essa variação. Os parâmetros Ti e Timax foram os que mais contribuíram para a variabilidade associada ao segundo eixo (CP2).

O experimento apresentou ótima repetibilidade e as amostras distinguiram-se umas das outras. A sacarose caracterizou-se pelos parâmetros Timax e Imax. A mistura ciclamato/sacarina 2:1 por Ti e o aspartame por Ttot, Tf, Área e Imax. A sucralose e a estévia não se caracterizaram por nenhum parâmetro especificamente.

Verificou-se correlação linear positiva (Tabela 5) entre os parâmetros Ttot e Imax, Área e Imax, Área e Ttot, Tf e Imax, Tf e Ttot e Tf e Área. 
TABELA 5 - VALORES DOS COEFICIENTES DE CORRELAÇÃO LINEAR DE PEARSON (R) ENTRE OS VETORES DA ANÁLISE DE COMPONENTES PRINCIPAIS PARA O ESTIIMULO SABOR DE FRUTA, EM SUCO DE MANGA A 8\% DE EQUIVALÊNCIA DE DOÇURA

\begin{tabular}{lcccccc}
\hline \multicolumn{1}{c}{ Vetores } & Imax & Timax & Ttot & Área & Ti & Tf \\
\hline Imax & 1,00000 & & & & & \\
Timax & 0,26762 & 1,00000 & & & & \\
Ttot & 0,92375 & $-0,00963$ & 1,00000 & & & \\
Área & 0,9675 & 0,20020 & 0,97167 & 1,00000 & & \\
Ti & 0,01162 & 0,57649 & $-0,11057$ & $-0,02316$ & 1,00000 & \\
Tf & 0,92706 & 0,00963 & 0,99945 & 0,97395 & $-0,07745$ & 1,00000 \\
\hline
\end{tabular}

Imax = intensidade máxima, Timax = tempo para atingir a intensidade máxima, Ttot $=$ tempo total de duração do estímulo, Área = área sob a curva, $\mathrm{Ti}=$ tempo de início da percepção do estímulo, Tf = tempo final da percepção do estímulo.

FIGURA 5 - GRÁFICO BIDIMENSIONAL DA ACP DOS PARÂMETROS DAS CURVAS TEMPO-INTENSIDADE PARA O ESTÍMULO SABOR DE FRUTA DAS AMOSTRAS DE SUCO DE MANGA, ADOÇADAS COM SACAROSE (SAC), CICLAMATO/SACARINA 2:1 (C/S 2:1), ASPARTAME (ASP), SUCRALOSE (SUC) E ESTÉVIA (EST) A 8\% DE EQUIVALÊNCIA DE DOÇURA

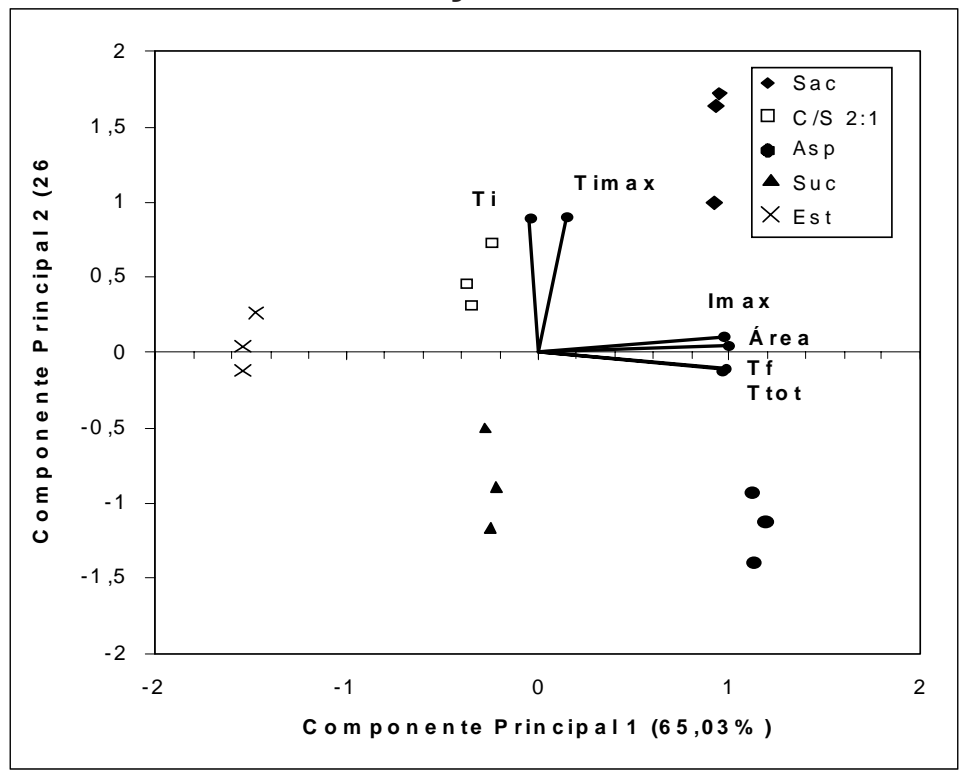


A análise de variância para o estímulo sabor de fruta em suco de manga evidenciou diferença significativa $(p<0,05)$ entre as amostras em relação a todos os parâmetros.

A Tabela 6 apresenta os resultados obtidos no teste de médias de Tukey.

TABELA 6 - MÉDIAS DOS PARÂMETROS DAS CURVAS TEMPOINTENSIDADE PARA O ESTÍMULO SABOR DE FRUTA DOS EDULCORANTES COM DOÇURA EQUIVALENTE A 8\% DE SACAROSE, ADICIONADOS EM SUCO DE MANGA

\begin{tabular}{ccccccc}
\hline Amostras & Imax & Timax & Ttot & Área & Ti & Tf \\
\hline Sac & $6,4944^{\mathrm{a}}$ & $11,5883^{\mathrm{a}}$ & $30,2629^{\mathrm{b}}$ & $108,411^{\mathrm{a}}$ & $2,4771^{\mathrm{a}}$ & $32,7400^{\mathrm{b}}$ \\
C/S & $5,4418^{\mathrm{d}}$ & $10,0300^{\mathrm{c}}$ & $26,9571^{\mathrm{c}}$ & $84,6190^{\mathrm{b}}$ & $2,5300^{\mathrm{a}}$ & $29,4871^{\mathrm{c}}$ \\
Asp & $6,2538^{\mathrm{b}}$ & $9,6333^{\mathrm{d}}$ & $33,1042^{\mathrm{a}}$ & $111,0330^{\mathrm{a}}$ & $2,2400^{\mathrm{b}}$ & $35,3442^{\mathrm{a}}$ \\
Suc & $5,7034^{\mathrm{c}}$ & $9,7654^{\mathrm{d}}$ & $26,7300^{\mathrm{c}}$ & $85,6760^{\mathrm{b}}$ & $2,2475^{\mathrm{b}}$ & $28,9775^{\mathrm{c}}$ \\
Est & $4,6199^{\mathrm{e}}$ & $10,3875^{\mathrm{b}}$ & $21,8971^{\mathrm{d}}$ & $66,7880^{\mathrm{c}}$ & $2,3396^{\mathrm{b}}$ & $24,2367^{\mathrm{d}}$ \\
\hline DMS & 0,0905 & 0,2071 & 0,8459 & 3,6244 & 0,1275 & 0,9251 \\
\hline
\end{tabular}

DMS = diferença mínima significativa obtida no teste de médias de Tukey.

Médias iguais na mesma coluna não diferem significativamente entre si $(p>0,05)$.

Imax = intensidade máxima, Timax = tempo para atingir a intensidade máxima, Ttot = tempo total de duração do estímulo, Área = área sob a curva, $\mathrm{Ti}=$ tempo de início da percepção do estímulo, $\mathrm{Tf}$ = tempo final da percepção do estímulo.

$\mathrm{SAC}=$ sacarose $, \mathrm{C} / \mathrm{S} 2: 1$ = ciclamato/sacarina, $\mathrm{Asp}=$ aspartame, $\mathrm{Suc}=$ sucralose Est $=$ estévia.

A maior Imax foi observada para a sacarose, seguida pelo aspartame, sucralose, mistura ciclamato/sacarina 2:1 e estévia, todas diferindo significativamente entre si.

A sacarose exibiu o maior Timax, seguida pelas amostras de estévia e mistura ciclamato/sacarina 2:1. O menor Timax foi verificado para o aspartame e para a sucralose que não diferiram entre si $(p>0,05)$.

Em relação aos parâmetros Ttot e Tf observou-se diferença significativa $(p<0,05)$ entre as amostras, exceto entre a sucralose e a mistura ciclamato/sacarina 2:1. As médias obedeceram a seguinte ordem decrescente: aspartame, sacarose, mistura ciclamato/sacarina 2:1, sucralose e estévia. 
A sacarose e o aspartame apresentaram as maiores médias para Área diferindo significativamente das outras amostras, mas não entre si. A menor média para este parâmetro foi observada para a estévia.

As amostras adoçadas com sacarose e com a mistura ciclamato/sacarina 2:1 apresentaram as maiores médias para Ti e diferiram significativamente das demais, que por sua vez não diferiram entre si.

A Figura 6 representa, graficamente, as médias dos parâmetros das curvas tempo-intensidade para o estímulo sabor de fruta, obtidas para os edulcorantes em suco de manga.

FIGURA 6 - CURVAS TEMPO-INTENSIDADE DO ESTÍMULO SABOR DE FRUTA PARA SACAROSE (SAC), CICLAMATO/ SACARINA 2:1 (C/S 2:1), ASPARTAME (ASP), SUCRALOSE (SUC) E ESTÉVIA (EST) A 8\% DE EQUIVALÊNCIA DE DOÇURA EM SUCO DE MANGA

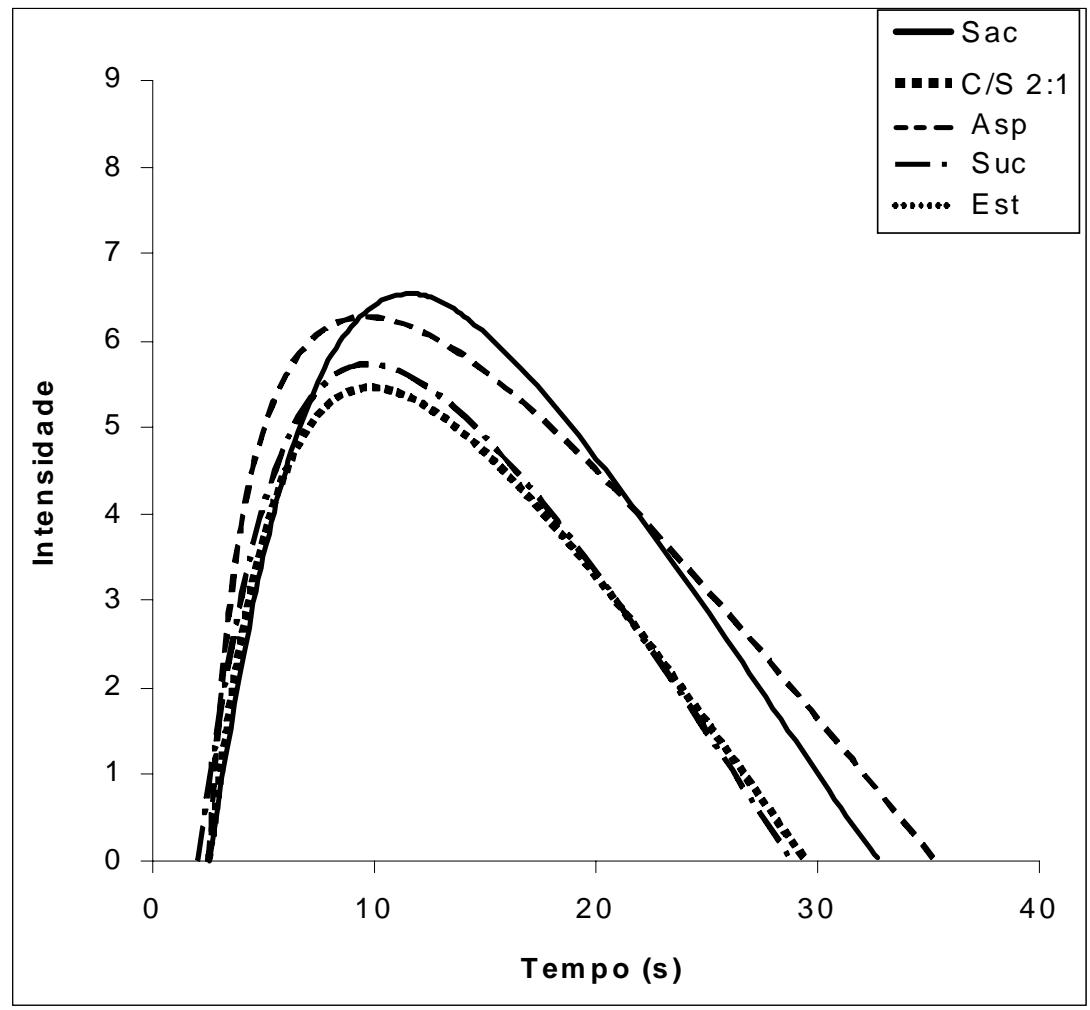


As amostras adoçadas com sacarose apresentaram a maior intensidade máxima (Imax) para o estímulo sabor de fruta, enquanto o aspartame exibiu o maior tempo total de duração do estímulo (Ttot) em suco de manga. Apesar da diferença de magnitude na percepção do estímulo, essa característica pode ser interpretada como intensificação do sabor de manga pelo aspartame em relação à sacarose. Outros estudos evidenciaram a capacidade do aspartame para intensificar o sabor de fruta em bebidas, seja em relação à intensidade ou em relação ao tempo de duração do estímulo. BALDWIN e KORSCHGEN (1979) estudaram o efeito do aspartame na intensificação do sabor de fruta em bebidas com sabor artificial de morango, cereja, laranja e em gelatinas. Observaram intensificação do sabor de fruta nas bebidas sabor cereja e laranja adoçadas com aspartame em comparação com as adoçadas com sacarose. Esse efeito não foi verificado nas bebidas sabor morango e nas gelatinas.

MATYSIAK e NOBLE (1991) avaliaram a doçura e o sabor de fruta em função do tempo em soluções aquosas com sabor artificial de laranja e adoçadas com aspartame, mistura aspartame/acessulfame-K e sacarose. O aspartame e a mistura aspartame/acessulfame-K exibiram tempo total de duração do estímulo (Ttot) superiores em relação aos estímulos doçura e sabor de fruta. Por outro lado, a estévia apresentou os menores valores de Imax e Ttot, provavelmente, devido à presença de amargor e de amargor residual que mascararam o sabor de fruta.

\section{CONCLUSÃO}

Nas curvas tempo-intensidade para o estímulo doce em suco de manga, a estévia apresentou médias superiores para todos os parâmetros exceto para intensidade máxima, indicando a presença de gosto residual doce pronunciado na amostra. A sacarose exibiu as menores médias para todos os parâmetros da curva tempo-intensidade, caracterizando-se pelo gosto doce limpo. O aspartame foi o edulcorante, cujo comportamento temporal mais se aproximou ao da sacarose quanto ao estímulo doce.

Em relação ao estímulo amargo, a sacarose, o aspartame e a sucralose apresentaram médias baixas para intensidade máxima, tempo total de duração do estímulo e área, evidenciando a presença de leve gosto amargo de curta duração. A mistura ciclamato/sacarina 2:1 caracterizouse pelo tempo de início da percepção do estímulo superior ao das demais 
amostras. A estévia apresentou a maior intensidade máxima para o estímulo amargo, que persistiu por longo período e confirmou a presença de amargor residual intenso na amostra.

Para o estímulo sabor de fruta, a sacarose exibiu a maior intensidade máxima e o aspartame o maior tempo total de duração do estímulo, indicando potencialização do sabor de fruta na amostra adoçada com esse edulcorante.

\section{Abstract}

COMPARISON OF TEMPORAL PERCEPTION OF SWEETENESS BITTERNESS AND FRUITINESS IN MANGO JUICE SWEETENED WITH SUCROSE, CYCLAMATE/SACCHARIN 2:1, ASPARTAME, SUCRALOSE AND STEVIOSIDE LEAF EXTRACT

Sweetness, bitterness and fruitiness were evaluated by time-intensity methodology for mango juice sweetened with four edulcorants (cyclamate/ saccharin 2:1, aspartame, sucralose and stevioside) and sucrose. The sample sweetened with stevioside showed maximal intensity for sweet and bitter tastes and these characteristic persisted for a long period, indicating intense sweet and bitter aftertaste. The sample with sucrose displayed a clean sweet taste with no unpleasant aftertaste. Sucrose had a higher intensity of fruit flavor. Although aspartame elicited significantly longer persistence of fruitiness, suggesting an intensification effect on fruitiness of mango juice. The sensorial profiles of aspartame were similar to that of sucrose in the timeintensity analysis.

KEY-WORDS: MANGO - JUICE; SENSORY ANALYSIS; SWEETENER; TIMEINTENSITY ANALYSIS.

\section{REFERÊNCIAS}

1 ABIAD. Associação Brasileira da Indústria de Alimentos Dietéticos e para Fins Especiais. Mercado Diet \& Light. Outubro 2004. Disponível em: <http://www.abiad.org.br/pdf/mercado diet light novo.pdf>. Acesso: 27 set. 2005.

2 AMERINE, M.A.; PANGBORN, R.M.; ROESSLER, E.B. Principles of sensory evaluation of food. New York: Academic Press, 1965. 602 p.

3 BALDWIN, R.E.; KORSCHGEN, B.M. Intensification of fruit flavors by aspartame. J. Food Sci., v.44, p.938-939, 1979. 
4 CARDELLO, H.M.A.B. Caracterização sensorial de aspartame, ciclamato/sacarina 2:1 e extrato de folhas de estévia (Stévia rebaudiana Bertoni): equivalências em doçura, análise descritiva quantitativa e análise tempo-intensidade. Campinas, 1996. 237 p. Tese (Doutorado em Tecnologia de Alimentos), Faculdade de Engenharia de Alimentos, Universidade Estadual de Campinas.

5 CARDELLO, H.M.A.B.; SILVA, M.A.A.P.; DAMÁSIO, M.H.; LOBÃO, F. Programa Sistema de Coleta de Dados Tempo-Intensidade. B. Soc. Bras. Ciênc. Tecnol. Alim., v.37, supl., p. 54-60, 2003.

6 CARDELLO, H.M.A.B.; SILVA, M. A.; DAMÁSIO, M.H. Análise tempointensidade dos estímulos doce e amargo de extrato de folhas de estévia (Stévia rebaudiana Bertoni) em doçura equivalente à sacarose em solução a 10\%. In: ALMEIDA, T.C.A.; HOUGH, G.; DAMÁSIO, M.H.; DA SILVA, M.A.A.P. Avanços em análise sensorial. São Paulo: Varella, 1999. p. 199-212.

7 CAVALLINI, D. C. U. Caracterização sensorial por análise descritiva quantitativa e análise tempo-intensidade de suco e de polpa de manga (Mangífera indica L.) adoçados com diferentes edulcorantes. Campinas, 2005. 190 p. Tese (Doutorado em Alimentos e Nutrição), Faculdade de Engenharia de Alimentos, Universidade Estadual de Campinas.

8 DAMÁSIO, M. H.; COSTELL, E. Análisis sensorial descriptivo: generación de descriptores y selección de catadores. R. Agroquím. Tecnol. Alim., v.31,n.2, p.165-178, 1991.

9 DUBOIS, G. E.; LEE, J. F. A simple technique for the evaluation of temporal taste properties. Chem. Senses, v.7, p.237-247, 1983.

10 HARRISON, S. K.; BERNHARDT, R. A. Time-intensity sensory characteristics of saccharin, xylitol and galactose, and their effect on the sweetness of lactose. J. Food Sci., v. 49, p.780-796, 1984.

11 IBGE. Instituto Brasileiro de Geografia e Estatística. Produção Agrícola Municipal 2003. Disponível em <http:// www.sidra.ibge.gov.br/bda/tabela/protablaspz $=\mathrm{t} 80=11>$. Acesso em: 27 set. 2005.

12 KEMP, S. E.; BIRCH, G. G. An intensity/time study of the taste of 
aminoacids. Chem. Senses, v.17, p.151-168, 1992.

13 KETELSEN, S. M.; KEAY, C. L.; WIET, S. G. Time-intensity parameters of selected carbohydrate and high potency sweeteners. J. Food Sci., v.58, p.1418-1421, 1993.

14 LARSON-POWERS, M.; PANGBORN, R. M. Pairs comparison and time-intensity measurements of sensory properties of beverages and gelatins containing sucrose or synthetic sweeteners. J. Food Sci., v. 43, p. $41-46,1978$.

15 LEE III, W. E.; PANGBORN, R. M. Time-intensity: the temporal aspects of sensory perception. Food Technol., v.40, p.71-82, 1986.

16 MATUSZEWSKA, I.; BARYLKO-PIKIELNA, N. The effect of sample exposure time on the time intensity response to $\mathrm{NaCl}$ solutions. Food Qual. Preference, v.6, p.43-48, 1995.

17 MATYSIAK, N. L.; NOBLE, A. C. Comparison of temporal perception of fruitness in model systems sweetened with aspartame, an aspartame+acessulfamer K blend, or sucrose. J. Food Sci., v.56, p. 823-826, 1991.

18 MEILGAARD, M.; CIVILLE, G. V.; CARR, B. T. Sensory evaluation techniques. $3^{\text {rd }}$ ed. New York: Boca Raton, 1999. 387 p.

19 MONTIJANO, H.; TOMÁS-BARBERÁN, F. A.; BORREGO, F. Propriedades tecnológicas y regulación de los edulcorantes de alta intensidade em la Unión Europea. Food Sci. Technol. International, v. 4, p.5-16, 1998.

20 NEILSON, A. J. Time-intensity studies. Drug Cosmet. Ind., v.80, p.452-454, 1957.

21 OTT, D. B.; EDWARDS, C. L.; PALMER, S. J. Perceived taste intensity and duration of nutritive and non-nutritive sweeteners in water using time-intensity (T-I) evaluations. J. Sensory Stud., v. 56, p. 535-542, 1991.

22 SAS institute. SAS User's Guide: statistics. Cary, 2001.

23 SEATON, L. New mango momentum. Fresh Produce Journal, v.15, 
p.37-39, 1996.

24 SWARTZ, M. Sensory screening of synthetic sweeteners using timeintensity evaluations. J. Food Sci., v.45, p.577-581, 1980.

25 VERMUNT, S. H. F.; PASMAN, W. J.; SCHAAFSMA, G.; KARDINAAL, A. F. M. Effects of sugar intake on body weight: a review. Obesity R., v.2, n.4, p.91-99, 2003.

25 WISEMAN, J. J.; McDANIEL, M. R. Modification of fruit flavors by aspartame and sucrose. J. of Food Sci., v.56, p.1668-1670, 1991. 